P-BR-094

BASIC RESEARCH

\section{Novel bioactive titanium membrane for bone regeneration; effect of alkali treatment}

\author{
Umehara H, Doi K, Kobatake R, Makihara Y, Oki Y, Kubo T, Tsuga K \\ Department of Advanced Prosthodontics, Hiroshima University \\ Graduate School of Biomedical \& Health Sciences
}

\section{Background and Aim}

\section{Background}

Guided bone regeneration (GBR) with barrier membrane technique is popular method of implant treatment for bone regeneration at bone defect site. Titanium is often used for barrier membrane, but titanium has no osteoinductive ability. Therefore, various modification methods have been applied to modify titanium ${ }^{1)}$. Our previous study demonstrated that alkali treatment creates nanoscale pore and titania layer on titanium thin membrane without mechanical strength reduction.

\section{Aim}

The aim of this study was to evaluate the ability of titanium membrane with alkali treatment to stimulate apatite-forming in simulated body fluid (SBF) and to promote bone regeneration in vivo.

\section{Methods and Materials}

\section{Materials}

- Titanium thin membrane

(pure titanium, thickness: $20 \mu \mathrm{m}$ )

- SBF: Hanks' Balanced Salt Solution

(HBSS, Lonza)

\section{Methods}

Membranes were washed in an ultrasonic cleaner with acetone and distilled water for 1 hour each.
Control group: no alkali treated titanium membrane
Alkali group: the titanium membranes were soaked in $5 \mathrm{~N}$ $\mathrm{NaOH}$ solution at $60{ }^{\circ} \mathrm{C}$ for $24 \mathrm{~h}$

\section{In vitro}

The samples were soaked in SBF for 7, 14 and 21 days.

- Surface structure: Scanning electron microscopy

- Surface composition: Electron probe microanalysis

\section{In vivo}

The critical-sized bone defect (diameter: $6 \mathrm{~mm}$ ) was trephined in the center of rat calvaria. The defect area was covered with the control membrane, alkali-treated membrane, or no membrane.

\section{4 weeks}

Four weeks later, tissue blocks were harvested.

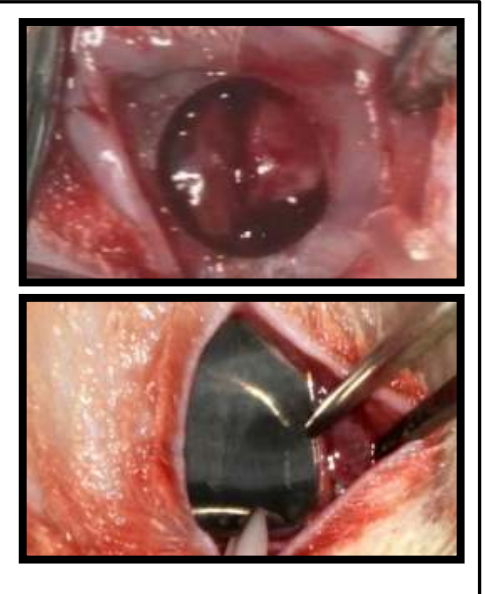

- Bone tissue area (BTA)

The area surrounding by a line connecting the upper end section and the lower end section of the original defect area

- Bone membrane contact ratio (BMC)

The length of the bone contact portion divided by the entire length of the membrane

\section{Results}

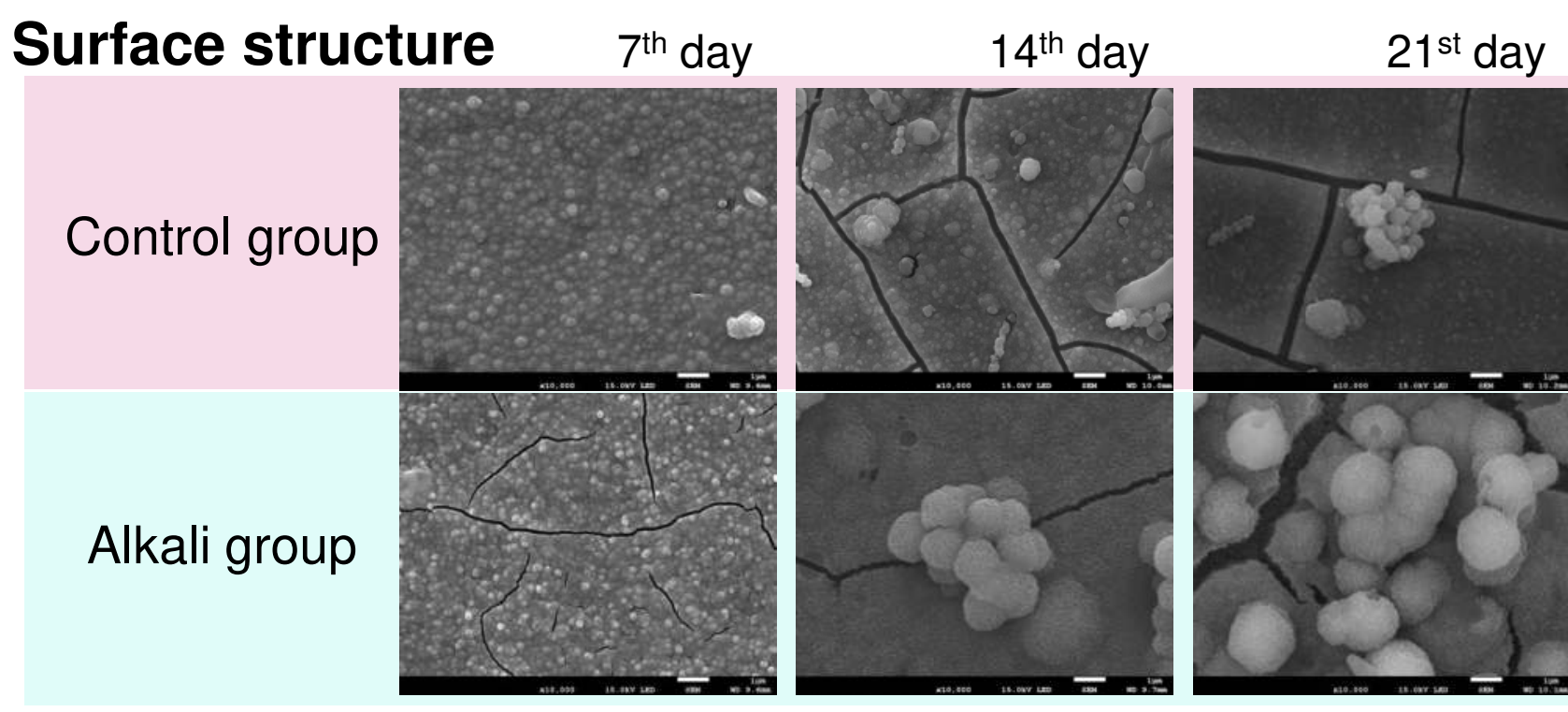

Surface composition

\begin{tabular}{|c|rrrrr|rrrrr|r|}
\cline { 2 - 12 } \multicolumn{1}{c|}{} & \multicolumn{5}{|c|}{ Control group } & \multicolumn{5}{c|}{ Alkali group } \\
\cline { 2 - 12 } \multicolumn{1}{c|}{} & $\mathrm{C}$ & $\mathrm{O}$ & \multicolumn{1}{c|}{$\mathrm{Ti}$} & $\mathrm{Ca}$ & $\mathrm{P}$ & $\mathrm{C}$ & $\mathrm{O}$ & $\mathrm{Ti}$ & $\mathrm{Ca}$ & $\mathrm{P}$ \\
\hline $0^{\text {th }}$ day & 0.26 & 5.91 & 93.8 & - & & 2.58 & 23.7 & 73.7 & 0.08 & \\
\hline $7^{\text {th }}$ day & 0.64 & 35 & 54.3 & 4.52 & 4.36 & 0.54 & 42.2 & 36.1 & 9.46 & 8.24 \\
\hline $14^{\text {th }}$ day & 0.65 & 42.8 & 40.4 & 7.52 & 7.09 & 0.71 & 43.9 & 32.8 & 10.3 & 8.83 \\
\hline $21^{\text {th }}$ day & 0.44 & 43.1 & 32.4 & 11 & 10.3 & 1.41 & 38.6 & 38.8 & 9.37 & 8.01 \\
\hline
\end{tabular}

Histological observation

Without membrane group

Control group

Alkali group

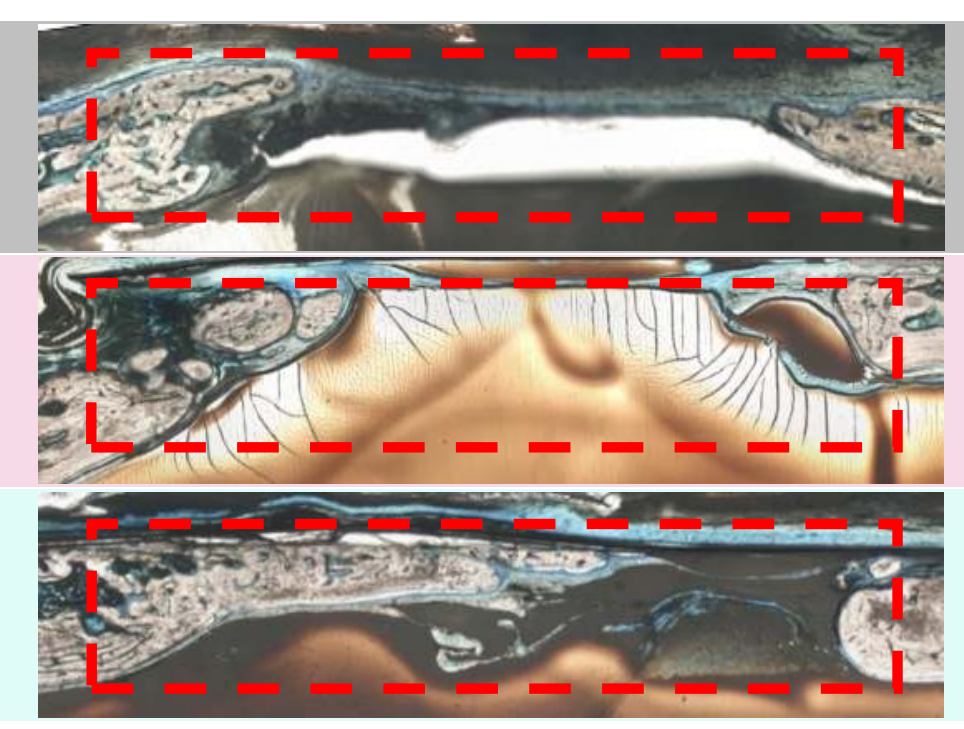

Histomorphometric analysis

\begin{tabular}{|l|cc|cr|}
\cline { 2 - 5 } \multicolumn{1}{c|}{} & \multicolumn{2}{c|}{ BTA $(\%)$} & \multicolumn{2}{c|}{ BMC $(\%)$} \\
\cline { 2 - 5 } \multicolumn{1}{c|}{} & total area & central area & total area & central area \\
\hline Control group & $35.1 \pm 20.6^{*}$ & $21.4 \pm 21.4$ & $21.6 \pm 12.1$ & $8.0 \pm 13.7$ \\
\hline Alkali group & $37.9 \pm 13.0^{* *}$ & $33.1 \pm 17.1^{* *}$ & $42.5 \pm 28.8$ & $41.1 \pm 29.3$ \\
\hline Without membrane & $11.7 \pm 4.6$ & $1.5 \pm 2.6$ & \multicolumn{1}{c}{} \\
\hline
\end{tabular}

Alkali-treated titanium membrane induces forming a layer of Ca-P composites on its surface rather than no alkali treated titanium surface. Alkali-treated titanium membranes exhibited a high apatite-forming ability in a body-simulating environment, and high bone forming ability.

\section{Conclusion}

Titanium membrane with alkali treatment stimulate apatiteforming in SBF and promote bone regeneration in vivo.

\section{Reference}

1) Umehara, H. Kobatake, R. Doi, K. Oki, Y. Makihara, Y. Kubo, T. Tsuga, K. Histological and Bone Morphometric Evaluation of Osseointegration Aspects by Alkali Hydrothermally-Treated. Implants. Appl Sci. 8, 635, 2017. 